\title{
Soliton Molecules and Full Symmetry Groups to the KdV-Sawada- Kotera-Ramani Equation
}

\author{
Na Xiong, ${ }^{1}$ Ya-Xuan Yu, ${ }^{2}$ and Biao Li ${ }^{2}{ }^{2}$ \\ ${ }^{1}$ College of Science and Technology, Ningbo University, Ningbo 315211, China \\ ${ }^{2}$ School of Mathematics and Statistics, Ningbo University, Ningbo 315211, China \\ Correspondence should be addressed to Biao Li; libiao@nbu.edu.cn
}

Received 9 February 2021; Revised 20 April 2021; Accepted 29 April 2021; Published 12 May 2021

Academic Editor: Leopoldo Greco

Copyright (C) $2021 \mathrm{Na}$ Xiong et al. This is an open access article distributed under the Creative Commons Attribution License, which permits unrestricted use, distribution, and reproduction in any medium, provided the original work is properly cited.

By $N$-soliton solutions and a velocity resonance mechanism, soliton molecules are constructed for the KdV-Sawada-Kotera-Ramani (KSKR) equation, which is used to simulate the resonances of solitons in one-dimensional space. An asymmetric soliton can be formed by adjusting the distance between two solitons of soliton molecule to small enough. The interactions among multiple soliton molecules for the equation are elastic. Then, full symmetry group is derived for the KSKR equation by the symmetry group direct method. From the full symmetry group, a general group invariant solution can be obtained from a known solution.

\section{Introduction}

Soliton molecules, also known as multisoliton complexes, are the bound states of solitons which exhibit molecule-like behavior [1]. Investigation on soliton molecules provides a direct route to study the interactions between solitary waves, and the formation and dissociation of soliton molecules are closely related to subjects such as soliton collision, soliton splashing, soliton rains, and the trapping of solitons. Besides, the significance they bring to the fundamental understanding of soliton physics, soliton molecules also present the possibility of transferring optical data surpassing the limitation of binary coding [2]. Recently, soliton molecules have been became one of the most challenging study field, which have been investigated theoretically and observed experimentally in some fields [3-8]. In 2005, soliton molecules were experimentally observed in dispersion-managed optical fibers [3]. In 2017, the evolution of femtosecond soliton molecules resolved in the cavity of a few-cycle mode-locked laser by means of an emerging timestretch technique [7]. In 2018, Liu et al. have experimentally observed the real-time dynamics of the entire buildup process of stable soliton molecules for the first time [8]. Two-soliton bound states in Bose-Einstein condensates with contact atomic interactions and some dynamic phenomena of soliton molecules were reported in $[9,10]$. In
2019, Lou [11] introduced a velocity resonant mechanism to form soliton molecules and asymmetric solitons for three-fifth order systems. Very recently, soliton molecules and some hybrid solutions involving Lump, breather, and positon have been investigated for some $(1+1)$-dimensional and $(2+1)$-dimensional equations by Hirota bilinear method and Darboux transformation [12-27].

As we all know, the study of symmetry is one of the most powerful methods for differential equations. The symmetry group direct method has been developed to obtain full symmetry groups for some PDEs [28-31]. Once the full symmetry group of a given system is given, the Lie point symmetry group can be derived, and sometimes, the discrete transformation group can also be expected. At the same time, the related Lie point symmetries can be recovered simply by restricting arbitrary functions or arbitrary constants in infinitesimal forms. Furthermore, one can reproduce a general group invariant solutions by the full symmetry groups and a known simple solution.

In this paper, we will investigate the following $\mathrm{KdV}$ Sawada-Kotera-Ramani equation [32-34]:

$$
u_{t}+a\left(3 u^{2}+u_{x x}\right)_{x}+b\left(15 u^{3}+15 u u_{x x}+u_{x x x x}\right)_{x}=0,
$$


which was proposed to describe the resonances of solitons in a one-dimensional space [32]. The conservation law for Eq. (1) was further given by Konno [34]. The KdVSawada-Kotera-Ramani equation (1) is a linear combination of the KdV equation and the Sawada-Kotera equation. When $b=0$, Eq. (1) is reduced to the KdV equation. When $a=0$, Eq. (1) is reduced to the Sawada-Kotera equation [35]. In Ref. [36], the authors investigated Lie symmetries, exact solutions, and integrability to the KdV-Sawada-Kotera-Ramani equation. To our knowledge, soliton molecules and full symmetry group of Eq. (1) have not been investigated so far.

The rest of paper is organized as follows. In Section 2, by introduce a velocity resonant condition, soliton molecules are constructed from $\mathrm{N}$-solitons of the KdV-SawadaKotera-Ramani equation. The transmission and collision properties of soliton molecules are discussed. In Section 3, the full symmetry group of the KdV-Sawada-Kotera-Ramani equation is derived by the symmetry group direct method. From the full symmetry group, a general group invariant solution can be obtained from a known solution. In Section 4 , short conclusions are given.

\section{Soliton Molecules}

The bilinear form of Eq. (1) is as follows

$$
\left(D_{x} D_{t}+a D_{x}^{4}+b D_{x}^{6}\right) f \cdot f=0
$$

under the transformation $u=2(\ln f)_{x x}$, where $D$ is the Hirota's bilinear differential operator and $f=f(x, t)$ is a real function of variables $\{x, t\}$. Based on the Hirota's bilinear theory, the $\mathrm{N}$-soliton solutions for Eq. (1) can be obtained as

$$
\begin{aligned}
& u=2(\ln f)_{x x}, \\
& f=\sum_{\rho=0,1} \exp \left(\sum_{1 \leq j<i \leq N}^{N} \rho_{i} \rho_{j} A_{i j}+\sum_{j=1}^{N} \rho_{j} \eta_{j}\right),
\end{aligned}
$$

with

$$
\begin{aligned}
\eta_{i} & =k_{i} x+w_{i} t+\phi_{i}, \\
e^{A_{i j}} & =-\frac{\left(k_{i}-k_{j}\right)\left(w_{i}-w_{j}\right)+a\left(k_{i}-k_{j}\right)^{4}+b\left(k_{i}-k_{j}\right)^{6}}{\left(k_{i}+k_{j}\right)\left(w_{i}+w_{j}\right)+a\left(k_{i}+k_{j}\right)^{4}+b\left(k_{i}+k_{j}\right)^{6}}, \\
w_{i} & =-\left(a k_{i}^{3}+b k_{i}^{5}\right), 1 \leq j<i \leq N,
\end{aligned}
$$

where $\left\{k_{i}, w_{i}, \phi_{i}\right\}(i=1,2, \cdots, N)$ are arbitrary constants, $\sum_{1 \leq j<i \leq N}^{N}$ is the summation of all possible pairs taken from $N$ elements with the condition $1 \leq j<i \leq N$, and $\sum_{\rho=0,1}$ indicates a summation over all possible combinations of $\rho_{i}, \rho_{j}=0,1(i, j=1,2, \cdots, N)$.

To find nonsingular analytical resonant excitation from Eq. (3), we apply the velocity resonance conditions $\left(k_{i} \neq \pm k_{j}, w_{i} \neq \pm w_{j}\right)$,

$$
\frac{k_{i}}{k_{j}}=\frac{w_{i}}{w_{j}}=\frac{b k_{i}^{5}+a k_{i}^{3}}{b k_{j}^{5}+a k_{j}^{3}} .
$$

Then, we can get the following expression

$$
\begin{aligned}
& k_{i}=\frac{\sqrt{-b\left(b k_{j}^{2}+a\right)}}{b}, \text { or, } \\
& k_{i}=-\frac{\sqrt{-b\left(b k_{j}^{2}+a\right)}}{b} .
\end{aligned}
$$

It can be known under the resonance condition (5) that two solitons, ith soliton and jth soliton in Eq. (3), exhibit one soliton molecule structure. To see this fact, we take $N=2$ as a simple example. When $N=2$, the solution (3) can be simplified to

$$
\begin{aligned}
u & =2\left[\ln \left(1+e^{\eta_{1}}+e^{\eta_{2}}+A_{12} e^{\eta_{1}+\eta_{2}}\right)\right]_{x x}, \\
\eta_{i} & =k_{i} x-\left(b k_{i}^{5}+a k_{i}^{3}\right) t+\phi_{i}, \\
A_{12} & =-\frac{\left(k_{1}-k_{2}\right)\left(-b k_{1}^{5}+b k_{2}^{5}-a k_{1}^{3}+a k_{2}^{3}\right)+a\left(k_{1}-k_{2}\right)^{4}+b\left(k_{1}-k_{2}\right)^{6}}{\left(k_{1}+k_{2}\right)\left(-b k_{1}^{5}-b k_{2}^{5}-a k_{1}^{3}-a k_{2}^{3}\right)+a\left(k_{1}+k_{2}\right)^{4}+b\left(k_{1}+k_{2}\right)^{6}} .
\end{aligned}
$$

Figure 1 displays the molecule structure expressed by Eq. (7) with the parameter selections

$$
\begin{aligned}
a & =6, \\
b & =-1, \\
k_{1} & =1, \\
k_{2} & =-\sqrt{5}, \\
\phi_{1} & =0, \\
\phi_{2} & =4 .
\end{aligned}
$$

From Figure 1, one can see that two solitons in the molecule are different because $k_{1} \neq k_{2}$ though the velocities of them are same.

If changing values $\phi_{1}$ and $\phi_{2}$, the distance between two solitons of the molecule will change, respectively. When the distance of two solitons is close enough to have an interaction with each other, the soliton molecule will become an asymmetric soliton. Figure 2 is the plots of the asymmetric soliton with parameters (8) except for $\left\{\phi_{1}=-8, \phi_{2}=10\right\}$. From Figure 2, one can see the twosoliton molecule keeps its asymmetric shape and velocity during the evolution.

Two-soliton molecules can be generated from four solitons; $k_{1}, w_{1}$ and $k_{2}, w_{2}$ satisfy Eq. (6); $k_{3}, w_{3}$ and $k_{4}, w_{4}$ satisfy Eq. (6) at the same time. Figure 3 displays the elastic 


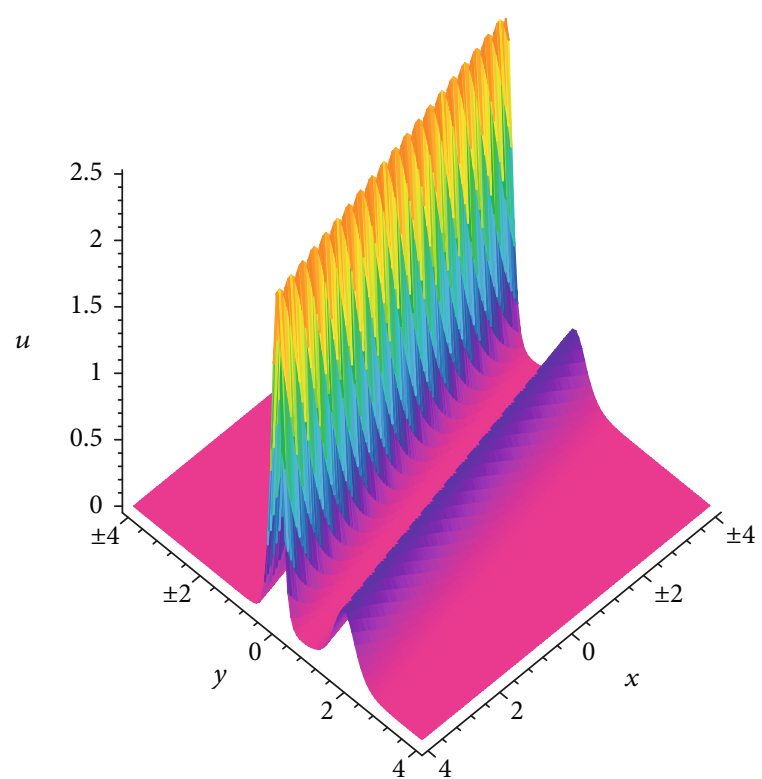

(a)

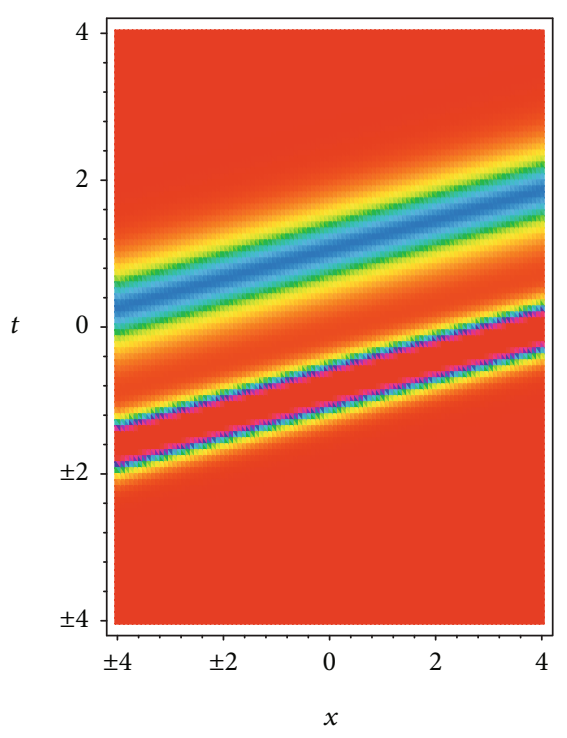

(b)

FIGURE 1: Soliton molecule structure for Eq. (1) with the parameter selections (8): (a) three-dimensional plot; (b) density plot.

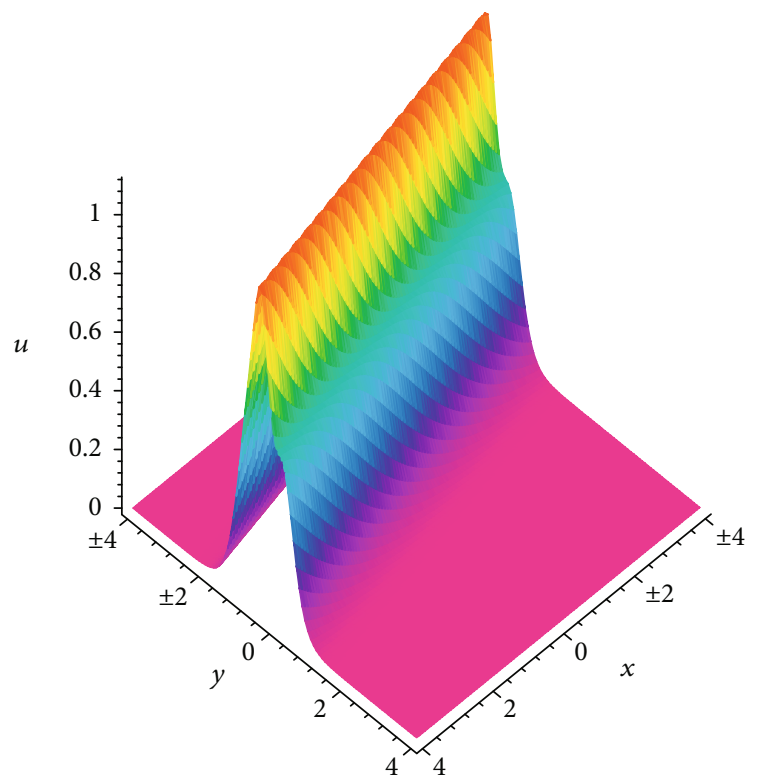

(a)

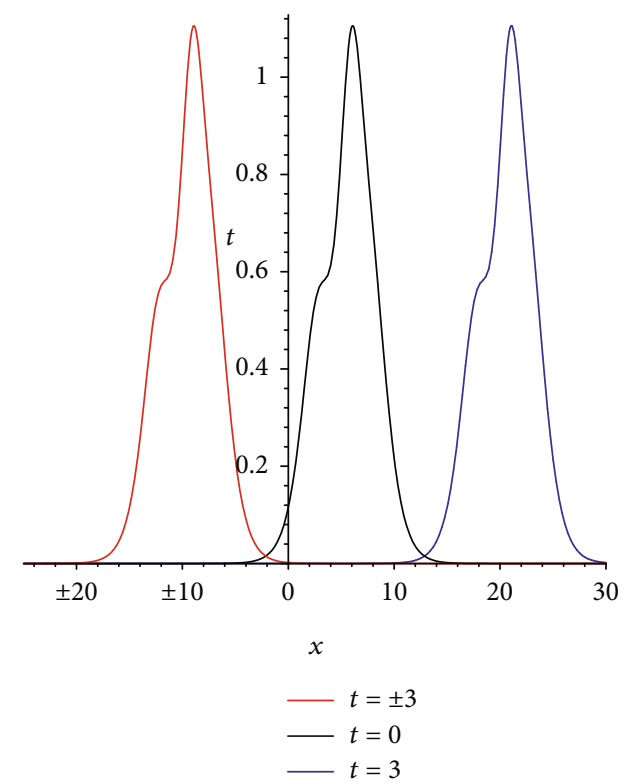

(b)

Figure 2: Asymmetric soliton for Eq. (1) with the parameter selections (8) except for $\left\{\phi_{1}=-8, \phi_{2}=10\right\}$ : (a) three-dimensional plot; (b) twodimensional plot at $t=-3,0,3$. 


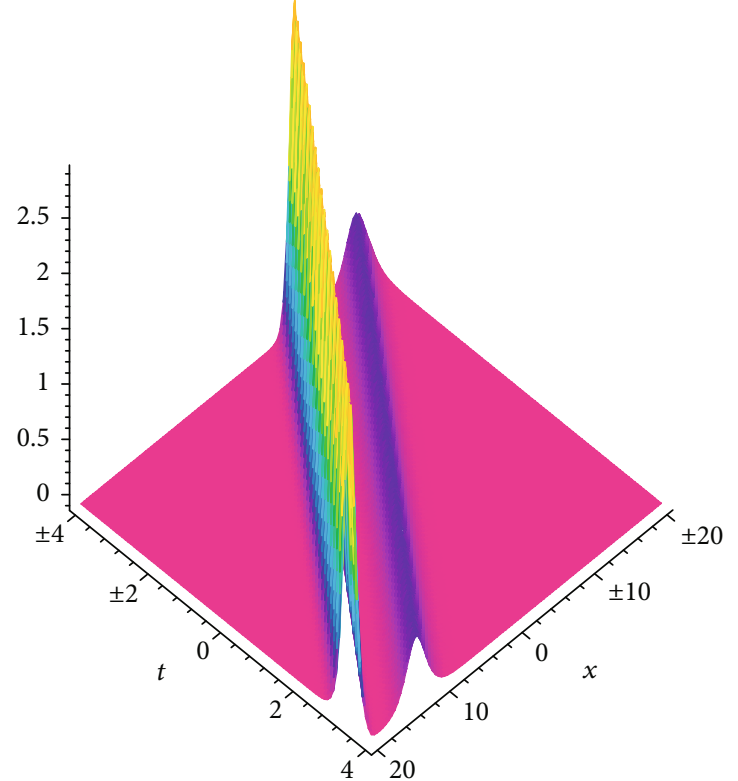

(a)

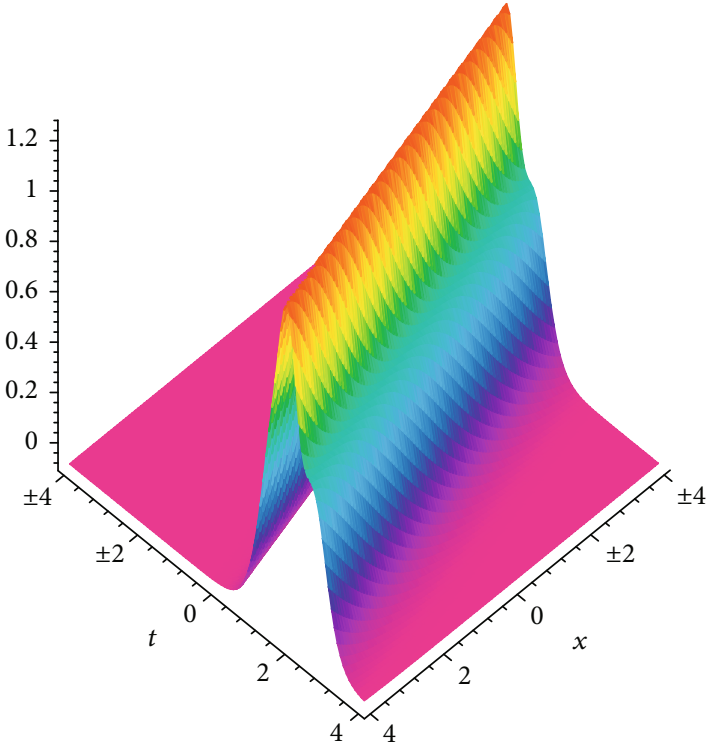

(b)

Figure 3: Three-dimensional plots of solution (17) with parameter selections (8) and (18): (a) soliton molecule structure; (b) asymmetric soliton structure.

interaction property for the solution (3) with $N=4$ and with parameter selections

$$
\begin{aligned}
a & =6, \\
b & =-1 \\
k_{1} & =1 \\
k_{2} & =-\sqrt{5} \\
\phi_{1} & =0 \\
\phi_{2} & =6 \\
k_{3} & =\frac{3}{4} \\
k_{4} & =-\frac{\sqrt{87}}{4}, \\
\phi_{3} & =4, \\
\phi_{4} & =8\left(k_{3}=\frac{1}{2}, k_{4}=-\frac{\sqrt{23}}{2}, \phi_{3}=-2, \phi_{4}=10\right) .
\end{aligned}
$$

From Figure 4, one can see that the height of wave peaks and velocities are not changed except for phase after collision.

\section{Finite Symmetry Groups}

According to the symmetry group direct method, we set the solutions of Eq. (1) as follows:

$$
u=\alpha+\beta U(\xi, \tau)
$$

where $\alpha=\alpha(x, t), \beta=\beta(x, t), \xi=\xi(x, t), \tau=\tau(x, t)$, and $U=$ $U(\xi, \tau)$ satisfy the same equations in Eq. (1)

$$
U_{\tau}+a\left(3 U^{2}+U_{\xi \xi}\right)_{\xi}+b\left(15 U^{3}+15 U U_{\xi \xi}+U_{\xi \xi \xi \xi}\right)_{\xi}=0
$$

Substituting Eq. (11) into Eq. (1) and eliminating all terms including $U_{\xi \xi \xi \xi \xi}$ by Eq. (11), we obtain one polynomial differential equations with respect to $U$ and their derivatives. Then, collecting the coefficients of $U$ and their derivatives, we obtain a set of overdetermined partial differential equations with respect to differential functions: $\{\alpha, \beta, \xi, \tau\}$. From the overdetermined PDEs, it is easy to find that

$$
\begin{aligned}
\tau & =\tau(t), \\
\beta & =\beta(t), \\
\xi_{x x} & =0, \\
\alpha_{x} & =0 .
\end{aligned}
$$

Now, the substitution of Eq. (12) into the overdetermined PDEs leads to

$$
\begin{aligned}
\beta\left(\tau_{t}-\xi_{x}^{5}\right) & =0 \\
\beta\left(45 \xi_{x} \alpha^{2} b+6 \xi_{x} \alpha a+\xi_{t}\right) & =0 \\
6 \beta \xi_{x}\left(-a \xi_{x}^{4}+15 b \alpha \beta+a \beta\right) & =0 \\
-\beta \xi_{x}^{3}\left(a \xi_{x}^{2}-15 b \alpha-a\right) & =0 \\
15 \beta \xi_{x}^{3} b\left(\beta-\xi_{x}^{2}\right) & =0 \\
45 b \beta \xi_{x}\left(\beta-\xi_{x}^{2}\right)\left(\beta+\xi_{x}^{2}\right) & =0 \\
\alpha_{t}=\beta_{t} & =0
\end{aligned}
$$




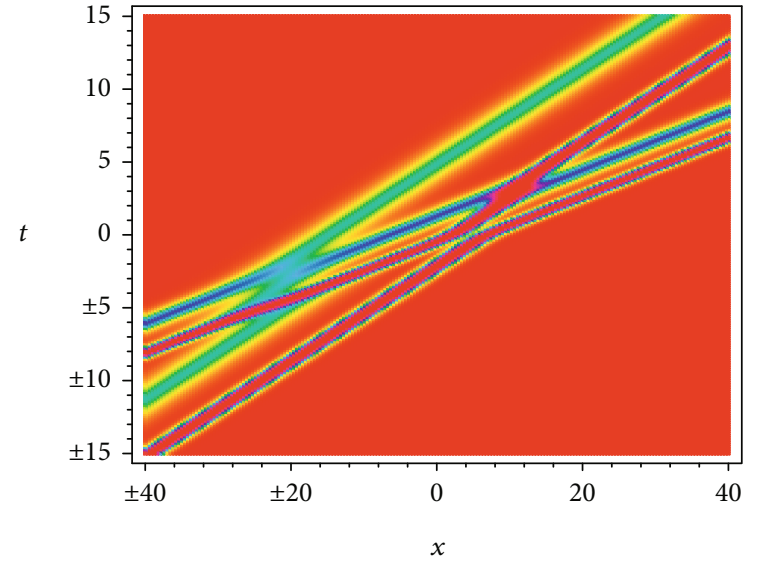

(a)

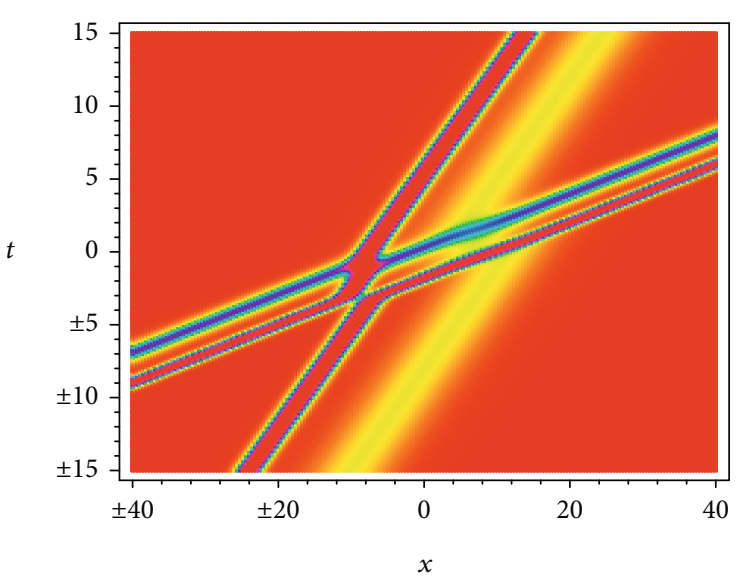

(b)

FIgURE 4: Two-soliton molecule structure for the solution (3) of Eq. (8) with the parameter selections (9).

With the help of symbolic computation after performing some calculations, the general solutions of Eqs. (12) and (13) are as follows

$$
\begin{aligned}
& \alpha=\frac{a\left(\sigma^{2} c^{2}-1\right)}{15 b}, \\
& \beta=c^{2} \sigma^{2}, \\
& \xi=\sigma c x-\frac{a^{2}\left(\sigma^{4} c^{5}-c\right)}{5 b} t+\xi_{0}, \tau=c^{5} t+\tau_{0},
\end{aligned}
$$

where $b \neq 0, c, \xi_{0}$, and $\tau_{0}$ are arbitrary constants while the constant $\sigma$ possess discrete values determined by

$$
\sigma^{5}=1 \Rightarrow\left\{\begin{array}{l}
\sigma_{1}=1, \\
\sigma_{2}=\frac{(\sqrt{5}-1)+i \sqrt{10+2 \sqrt{5}}}{4}, \\
\sigma_{3}=\frac{(\sqrt{5}-1)-i \sqrt{10+2 \sqrt{5}}}{4}, \\
\sigma_{4}=\frac{(\sqrt{5}-1)+i \sqrt{10-2 \sqrt{5}}}{4}, \\
\sigma_{5}=\frac{(\sqrt{5}-1)-i \sqrt{10+2 \sqrt{5}}}{4},(i=\sqrt{-1}) .
\end{array}\right.
$$

From the above results, one can get the following symmetry group theorem for Eq. (1).

Theorem 1. If $U=U(x, t)$ is a solution of KdV-SawadaKotera-Ramani equation (1), then so is

$$
u=\frac{a\left(\sigma^{2} c^{2}-1\right)}{15 b}+\sigma^{2} c^{2} U(\xi, \tau)
$$

with (14) and (15).
From the symmetry group Theorem 1, we know that for the real KSKR equation, the Lie point symmetry group which corresponds to $\sigma=1$. For the complex KSKR equation, the symmetry group is divied into five sectors which correspond to five values of $\sigma$ in Eq. (15). At the same time, we can derive the classical Lie symmetry from Theorem 1 by taking arbitrary constants $\left\{c, \xi_{0}, \tau_{0}\right\}$ as some special infinitesimal parameter forms.

Furthermore, one can obtain a general group invariant solutions of KSKR equation by Theorem 1 and a known simple solution. For example, from Eq. (7) and Theorem 1, we can derive a general two solitons for KSKR equation as follows

$$
\begin{aligned}
& u=\frac{a\left(\sigma^{2} c^{2}-1\right)}{15 b}+2 \sigma^{2} c^{2}\left[\ln \left(1+e^{\eta_{1}}+e^{\eta_{2}}+A_{12} e^{\eta_{1}+\eta_{2}}\right)\right]_{\xi \xi}, \\
& \eta_{i}=k_{i} \xi-\left(b k_{i}^{5}+a k_{i}^{3}\right) \tau+\phi_{i},
\end{aligned}
$$

with (14) and (15).

It is necessary to point out that when two-soliton solution (17) exhibits one soliton molecule structure, the velocity resonance condition is the same as (5). Figure 3 displays the molecule structure and asymmetric soliton structure expressed by (17) with the parameter selections (8) and

$$
\begin{aligned}
\sigma & =1, \\
c & =1.1, \\
\xi_{0} & =2\left(\xi_{0}=0\right), \\
\tau_{0} & =1\left(\tau_{0}=-2\right) .
\end{aligned}
$$

\section{Conclusion}

In this paper, we investigated the KdV-Sawada-KoteraRamani (KSKR) equation, which is used to simulate the resonances of solitons in one-dimensional space. On the basis of general $\mathrm{N}$-soliton express and velocity resonance mechanism, we obtained one soliton molecule and multiple soliton 
molecule structure for the KSKR equation. Asymmetric soliton can be formed by adjusting the distance between two solitons to small enough. The interactions among these soliton molecules for the KSKR equation are elastic. Then, we derived the full symmetry group for the KSKR equation by the symmetry group direct method. From the full symmetry group, a general group invariant solution can be obtained from a known solution. The results of the paper can be expected to provide some useful information for the dynamic behaviors of KSKR equations. It is necessary to note that on the basis of $\mathrm{N}$-soliton solutions by Hirota bilinear method, we only obtain a soliton molecule including two solitons for the KSKR equation. It is usually not easy to derive a soliton molecule containing multiple solitons. In order to obtain a soliton molecule containing multiple solitons, one can investigate $N$-solitons from Darboux transform to find a soliton molecule with multiple solitons [14]. The method to construct soliton molecules and the symmetry group method can be applied to investigate other nonlinear models.

\section{Data Availability}

No data were used to support this study.

\section{Conflicts of Interest}

The authors declare that they have no conflicts of interest.

\section{Acknowledgments}

This work is supported by the National Natural Science Foundation of China under Grant Nos. 11805106, 11775121, and 11435005 and K.C.Wong Magna Fund in Ningbo University.

\section{References}

[1] N. Akhmediev and A. Ankiewicz, "Multi-soliton complexes," Chaos: An Interdisciplinary Journal of Nonlinear Science, vol. 10, no. 3, article 600, 2000.

[2] W. Weng, R. Bouchand, E. Lucas, E. Obrzud, T. Herr, and T. J. Kippenberg, "Heteronuclear soliton molecules in optical microresonators," Nature Communications, vol. 11, no. 1, article 2402, 2020.

[3] M. Stratmann, T. Pagel, and F. Mitschke, "Experimental observation of temporal soliton molecules," Physical Review Letters, vol. 95, article 143902, 2005.

[4] A. Hause, H. Hartwig, B. Seifert, H. Stolz, M. Böhm, and F. Mitschke, "Phase structure of soliton molecules," Physical Review A, vol. 75, article 063836, 2007.

[5] K. Krupa, K. Nithyanandan, U. Andral, P. Tchofo-Dinda, and P. Grelu, "Real-time observation of internal motion within ultrafast dissipative optical soliton molecules," Physical Review Letters, vol. 118, article 243901, 2017.

[6] L. Li, H. T. Huang, L. Su et al., "Various soliton molecules in fiber systems," Applied Optics, vol. 58, no. 10, pp. 2745-2753, 2019.

[7] G. Herink, F. Kurtz, B. Jalali, D. R. Solli, and C. Ropers, "Realtime spectral interferometry probes the internal dynamics of femtosecond soliton molecules," Science, vol. 356, pp. 50-54, 2017.
[8] X. M. Liu, X. K. Yao, and Y. D. Cui, "Real-time observation of the buildup of soliton molecules," Physical Review Letters, vol. 121, article 023905, 2018.

[9] U. Al Khawaja and H. T. C. Stoof, "Formation of matter-wave soliton molecules," New Journal of Physics, vol. 13, article 085003, 2011.

[10] S. M. Almarzoug, S. M. Alamoudi, U. Al Khawaja, H. Bahlouli, and B. B. Baizakov, "Scattering of a matter-wave single soliton and a two-soliton molecule by an attractive potential," Physical Review E, vol. 83, no. 2, article 026603, 2011.

[11] S. Y. Lou, "Soliton molecules and asymmetric solitons in three fifth order systems via velocity resonance," Journal of Physics Communications, vol. 4, article 041002, 2020.

[12] X. B. Wang, S. F. Tian, C. Y. Qin, and T. T. Zhang, "Dynamics of the breathers, rogue waves and solitary waves in the $(2+1)$ dimensional Ito equation," Applied Mathematics Letters, vol. 68, pp. 40-47, 2017.

[13] W. T. Li, Z. Zhang, X. Y. Yang, and B. Li, "High-order breathers, lumps and hybrid solutions to the $(2+1)$-dimensional fifth-order $\mathrm{KdV}$ equation," International Journal of Modern Physics B, vol. 33, no. 22, p. 1950255, 2019.

[14] Z. Zhang, X. Yang, and B. Li, "Soliton molecules and novel smooth positons for the complex modified KdV equation," Applied Mathematics Letters, vol. 103, article 106168, 2020.

[15] S. Yang, Z. Zhang, and B. Li, "Soliton molecules and some novel types of hybrid solutions to $(2+1)$-dimensional variable-coefficient Caudrey-Dodd-Gibbon-Kotera-Sawada equation," Advances in Mathematical Physics, vol. 2020, 9 pages, 2020.

[16] J. J. Dong, B. Li, and M. Yuen, "Soliton molecules and mixed solutions of the $(2+1)$-dimensional bidirectional SawadaKotera equation," Communications in Theoretical Physics, vol. 72, article 025002, 2020.

[17] D. H. Xu and S. Y. Lou, "Dark soliton molecules in nonlinear optics," Acta Physica Sinica, vol. 69, article 014208, 2020(in Chinese).

[18] Z. W. Yan and S. Y. Lou, "Soliton molecules in Sharma-TassoOlver-Burgers equation," Applied Mathematics Letters, vol. 104, article 106271, 2020.

[19] B. Ren, J. Lin, and Z. M. Lou, "Consistent Riccati expansion and rational solutions of the Drinfel'd-Sokolov-Wilson equation," Applied Mathematics Letters, vol. 105, article 106326, 2020.

[20] X. W. Jin and J. Lin, "Rogue wave, interaction solutions to the KMM system," Journal of Magnetism and Magnetic Materials, vol. 502, article 166590, 2020.

[21] B. Ren and J. Lin, "Soliton molecules, nonlocal symmetry and CRE method of the KdV equation with higher-order corrections," Physica Scripta, vol. 95, article 075202, 2020.

[22] B. Ren, W. X. Ma, and J. Yu, "Characteristics and interactions of solitary and lump waves of a (2+1)-dimensional coupled nonlinear partial differential equation," Nonlinear Dynamics, vol. 96, pp. 717-727, 2019.

[23] L. Kaur and A. M. Wazwaz, "Lump, breather and solitary wave solutions to new reduced form of the generalized BKP equation," International Journal of Numerical Methods for Heat \& Fluid Flow, vol. 29, pp. 569-579, 2019.

[24] L. Kaur and A. M. Wazwaz, "Bright-dark lump wave solutions for a new form of the (3+1)-dimensional KPBoussinesq equation," Romanian Reports in Physics, vol. 71, p. 102, 2019. 
[25] L. Kaur and A. M. Wazwaz, "Dynamical analysis of lump solutions for (3+1)-dimensional generalized KP-Boussinesq equation and its dimensionally reduced equations," Physica Scripta, vol. 93, article 075203, 2018

[26] L. Kaur and A. M. Wazwaz, "Bright - dark optical solitons for Schrödinger-Hirota equation with variable coefficients," Optik, vol. 179, pp. 479-484, 2019.

[27] S. Singh, L. Kaur, K. Sakkaravarthi, R. Sakthivel, and K. Murugesan, "Dynamics of higher-order bright and dark rogue waves in a new (2+1)-dimensional integrable Boussinesq model," Physica Scripta, vol. 95, article 115213, 2020.

[28] S. Y. Lou and H. C. Ma, "Non-Lie symmetry groups of $(2+1)-$ dimensional nonlinear systems obtained from a simple direct method," Journal of Physics A-Mathematical and General, vol. 38, no. 7, pp. L129-L137, 2005.

[29] B. Li, W. C. Ye, and Y. Chen, "Symmetry, full symmetry groups, and some exact solutions to a generalized DaveyStewartson system," Journal of Mathematical Physics, vol. 49, article 103503, 2008.

[30] B. Li, Y. Q. Li, and Y. Chen, "Finite symmetry transformation groups and some exact solutions to $(2+1)$-dimensional cubic nonlinear Schrödinger equantion," Communications in Theoretical Physics, vol. 51, pp. 773-776, 2009.

[31] H. P. Zhang, B. Li, and Y. Chen, "Full symmetry groups, Painlevé integrability and exact solutions of the nonisospectral BKP equation," Applied Mathematics and Computation, vol. 217, no. 4, pp. 1555-1560, 2010.

[32] R. Hirota and M. Ito, "Resonance of solitons in one dimension," Journal of The Physcicla Society of Japan, vol. 52, no. 3, pp. 744-748, 1983.

[33] J. Yang, "Dynamics of embedded solitons in the extended Korteweg-de Vries equations," Studies in Applied Mathematics, vol. 106, no. 3, pp. 337-365, 2001.

[34] K. Konno, "Conservation laws of modified Sawada-Kotera equation in complex plane," Journal of The Physcicla Society of Japan, vol. 61, no. 1, pp. 51-54, 1992.

[35] K. Sawada and T. Kotera, "A method for finding N-soliton solutions of the K.d.V. equation and K.d.V.-like equation," Progress of Theoretical Physics, vol. 51, pp. 1355-1367, 1974.

[36] P. L. Ma, S. F. Tian, T. T. Zhang, and X. Y. Zhang, "On Lie symmetries, exact solutions and integrability to the KdVSawada-Kotera-Ramani equation," The European Physical Journal Plus, vol. 131, pp. 1-15, 2016. 